Recebido em 04/2019. Aceito para publicação em 04/2019.

\title{
AVALIAÇÃO DO PROTOCOLO DE VELOCIDADE DE NADO PARA 4 MMOL DE LACTATO (PROTOCOLO DO V4) PARA NADADORES
}

\section{EVALUATION OF THE PROTOCOL FOR SWIMMING VELOCITY FOR 4 MMOL OF LACTATE (V4 PROTOCOL) FOR SWIMMERS}

\author{
Marcelo Celestino Vaccari ${ }^{1}$ \\ Patrícia Sardinha Leonardo Lopes Martins ${ }^{2}$ \\ Mário Oliveira Lima ${ }^{3}$ \\ Rodrigo Alvaro Brandão Lopes Martins ${ }^{4}$
}

Resumo: Uma questão primária para técnicos e cientistas do esporte é como prescrever um programa de treinamento obtendo o máximo de desempenho com um mínimo de prejuízos para o atleta. Métodos para a determinação do Limiar Anaeróbio utilizam tanto protocolos diretos quanto indiretos. Neste trabalho visamos realizar uma análise prática do protocolo do V4 em nadadores velocistas e fundistas. $O$ estudo desenvolveu-se com a equipe de natação FADENP de São José dos Campos/SP com 19 nadadores (1630 anos, 8 do sexo feminino e 11 do sexo masculino). Nadadores velocistas realizaram 24 seqüências de $100 \mathrm{~m}$, com intervalos de 30 segundos. Nadadores fundistas realizaram 6 seqüências de $400 \mathrm{~m}$. Todos os testes foram realizados na mesma piscina de 25 metros. Os atletas foram capazes de desenvolver a velocidade de natação preconizada no pré-teste tanto os nadadores velocistas quanto para fundistas. Não houve diferenças significativas para as concentrações de lactato plasmático nos atletas velocistas, No entanto, quanto aos nadadores fundistas, não foram alcançadas as concentrações de lactato preconizadas. Em nosso trabalho e sob nossas condições experimentais podemos concluir que, ao menos para nadadores velocistas os parâmetros preconizados pelo teste do V4 foram altamente fidedignos.

Palavras-chave: Lactato; natação; protocolo V4.

Abstract: One of the main questions for coaches and sports scientists is how to prescribe a training program to achieve maximal performance without problems to the athlete. In this paper our purpose is to perform a practical evaluation of the V4 protocol for short- as well as for long-distance swimmers. The study was developed with the group of swimmers of FADENP from São José dos Campos/SP, consisting in 19 swimmers (16-30 years old, 8 females and 11 males). Short-distance swimmers were submitted to 24 sequences of $100 \mathrm{~m}$, with 30-second intervals. Long-distance swimmers were submitted to 6 sequences of $400 \mathrm{~m}$. All tests were performed at the same 25-m swimming pool. All athletes were able to develop the swimming velocity determined by the pre-tests. There was no significant difference between the predetermined and obtained lactate concentration for short-distance swimmers. However, regarding the longdistance swimmers, the obtained lactate concentration was significantly lower than that pre-determined by the protocol. In this paper, at least in our experimental conditions, we can conclude that for short-distance swimmers, the V4 protocol was very accurate and reproducible.

Keywords: Lactate; swimming; V4 protocol.

\footnotetext{
${ }^{1}$ Mestre em Ciências Biológicas - Técnico Nivel 1 de Natação - CBDA, Minas Tênis Clube, MG, Brasil. E-mail: vaccari@minastc.com.br.

2 Doutora em Engenharia Biomédica, Supervisora de Ortopedia da UNIVAP. São José dos Campos/SP, Brasil. Email: pssardinha@yahoo.com.br.

3 Doutor em Engenharia Biomédica, Supervisor de Neurologia e Professor do PPG em Engenharia Biomédica da Universidade do Vale do Paraíba - Univap. São José dos Campos/SP, Brasil. E-mail: mol@univap.br.

4 Prof. Livre-Docente em Farmacologia, Coordenador do Mestrado em Engenharia Biomédica da Universidade do Vale do Paraíba - Univap, São José dos Campos/SP, Brasil. E-mail: rodrigo@univap.br.
} 


\section{INTRODUÇÂO}

Um desempenho competitivo de sucesso na modalidade esportiva de natação requer que um nadador talentoso alcance um alto nível técnico e físico, e que seu desempenho competitivo seja reprodutível, tanto em treinamentos quanto em competições. A técnica e o condicionamento devem ser sustentados por um alicerce psicológico sólido, uma estratégia tática apropriada, e um organismo saudável (OLBRECHT, 2000).

O caminho para o sucesso em um meio olímpico leva entre 6 a 16 anos de treinamento estruturado por um técnico especializado, que utiliza intuição, experiência e conhecimento científico. A chave para o sucesso não se encontra somente no treinamento pesado, mas sim em um treinamento estudado científica e cuidadosamente. Isto requer que o treinamento de um nadador de alto nível seja planejado e monitorado, e que seu desempenho competitivo seja avaliado, não somente pelo tempo desenvolvido, mas também com respeito a técnica e estratégia.

Dentre os componentes que afetam o desempenho do nadador, podemos destacar a velocidade, mecanismos de respiração, técnicas de largada e virada, potência e capacidade anaeróbia, força muscular, flexibilidade e ainda, fatores psicológicos. A análise conjunta destes fatores é de vital importância para a melhoria do desempenho de um atleta de alto nível (SMITH; NORRIS; HOGG, 2002).

O Treinamento físico visa aprimorar as capacidades motoras específicas utilizadas no esporte, minimizando as dificuldades encontradas na realização dos movimentos e a manutenção do desempenho durante as temporadas de trabalho e período competitivo. É um processo que consiste em uma série de estresses fisiológicos que produzem ou preservam adaptações específicas com o objetivo de aumentar a habilidade do sujeito na tolerância dos fatores estressantes surgidos com o treinamento (GOLLNICK et al, 1990; COYLE; HOPPER; COGGAN, 1990).

Uma questão primária para técnicos e cientistas do esporte é como prescrever um programa de treinamento obtendo o máximo de desempenho com um mínimo de prejuízos para o atleta (HAWLEY; STEPTO, 2001). Considerando os princípios da especificidade, sobrecarga progressiva e recuperação, o somatório destes elementos são estímulos que podem resultar tanto numa melhora quanto na diminuição do desempenho, caracterizado por Morton (1997), respectivamente como estado de fitness ou fadiga. Segundo o autor, em algum ponto está a quantidade ideal de treinamento que resultará no desempenho, estabelecendo o limiar entre o fitness e a fadiga.

Segundo Hay e Guimarães (1983), a natação competitiva pode ser dividida em três elementos principais: largada, virada e limpeza, e velocidade final nos últimos 5 $20 \mathrm{~m}$. Em 1988, estes elementos foram utilizados para a análise das competições préolímpicas (NELSON; BROWN; KENNEDY, 1990). Além disso, a freqüência de 
braçadas, distância de braçadas e velocidade ao meio da piscina foram utilizados nos Jogos Olímpicos de Seul em 1988. Atualmente, a utilização de técnicas mais modernas, como a vídeo-documentação e análise, assim como, o monitoramento de variáveis bioquímicas para avaliação de desempenho e planejamento do treinamento têm sido fundamentais para equipes de alto nível (SMITH; NORRIS; HOGG, 2002).

A análise acurada e 0 acesso a vários componentes relacionados ao desempenho do atleta dentro de um contexto do treinamento é um importante processo para os técnicos e fisiologistas do esporte incluírem como aspectos fundamentais para os programas de treinamento e competição de nadadores (TROUP, 1986; PANSOLD; ZINNER, 1991; PYNE; MAW; GOLDSMITH, 2000).

Vários testes não invasivos têm sido discutidos na literatura como, por exemplo, os de 30 e 60 minutos de natação (OLBRECHT et al, 1985), e ainda os de 2000 e 3000 metros (MADSEN, 1982). Todos eles fornecem, supostamente, uma interpretação da capacidade aeróbia de resistência. Tais testes são baseados na interrelação entre o consumo de oxigênio $\left(\mathrm{VO}_{2}\right)$, a concentração de lactato plasmático e a velocidade de natação. Existe, principalmente, um grande número de trabalhos considerando a concentração de lactato plasmático como o principal indicador do grau de esforço ou impacto sobre o nadador durante o treinamento ou competição (TROUP, 1986; PANSOLD; ZINNER, 1991; PYNE; MAW; GOLDSMITH, 2000). No entanto, as dificuldades de padronização de testes específicos para avaliação e monitorização de desempenho em nadadores, assim como, a definição de parâmetros a serem monitorados, tem representado uma difícil barreira a ser transposta. Há de se convir, que os testes ergométricos ou ergoespirométricos tradicionais em esteira rolante ou bicicletas estacionárias não são exatamente os mais adequados para avaliação de atletas de natação. Neste contexto, a caracterização e o estabelecimento de novas metodologias de avaliação para atletas de natação assumem fundamental importância para o desenvolvimento do desporto, e para o conhecimento de aspectos fisiológicos específicos da atividade de natação.

A resposta do lactato sanguíneo ao exercício tem sido utilizada para a avaliação da capacidade aeróbica de indivíduos sedentários, ativos, assim como de atletas de competição (WELTMAN, 1995). Tratando-se de um parâmetro sub-máximo, a resposta do lactato sangüíneo ao exercício é considerada mais apropriada do que a captação máxima de oxigênio $\left(\mathrm{VO}_{2 \max }\right)$, para a avaliação dos efeitos do treinamento e, devido a sua forte correlação com o desempenho de resistência, pode ser utilizada para a prescrição individual de intensidades de treinamento (SJODIN; JACOBS; SVENDENHAG, 1982; COYLE; HOPPER; COGGAN, 1990).

A terminologia mais comum utilizada para se descrever a resposta do lactato sangüíneo durante o exercício é o "Limiar Anaeróbico". Este representa a maior intensidade de exercício no qual o balanço entre a produção e a remoção de lactato ocorre (HECK et al, 1985). 
Métodos para a determinação do Limiar Anaeróbio utilizam tanto protocolos diretos quanto indiretos são utilizados onde concentrações fixas de lactato sangüíneo (4 mmol. I $^{-1}$ ) ou variáveis (STEGMAN; KINDERMANN; SCHNABEL, 1981; SJODIN; JACOBS; SVENDENHAG, 1982; TEGTBUR; BUSSE; BRAUMANN, 1993) são utilizados. Na natação, a determinação da velocidade de nado capaz de gerar uma concentração sangüínea de lactato de $4 \mathrm{mmol.}^{-1}$, utilizando o conceito de "Velocidade Crítica", tem sido proposto como uma maneira indireta de se avaliar este parâmetro, uma vez que nem sempre é possível ou conveniente se determinar esta velocidade nesta concentração através da análise sangüínea de lactato (WAKAYOSHI et al, 1992). Este método é pois, prático e aplicável, e permite sua utilização em maior número de atletas (DENADAI; GRECO; TEIXEIRA, 2000).

Wakayoshi e colaboradores (1992) definiram Velocidade Crítica como a velocidade de nado que, teoricamente, poderia ser mantida sem exaustão. A Velocidade Crítica foi então expressa como a inclinação curva que relaciona a distância percorrida em cada uma de seis velocidades e distâncias pré-determinadas. No entanto, poucos autores têm se dedicado a investigar e mesmo confirmar estes resultados encontrados por Wakayoshi et al. (1992). Denadai, Greco e Teixeira (2000) investigaram a teoria da Velocidade Crítica da natação em crianças, e concluíram que a Velocidade Crítica, na verdade, subestima a intensidade de natação correspondente a concentração sanguínea de lactato de $4 \mathrm{mmol}^{-\mathrm{I}^{-1}}$ no grupo estudado, de crianças de 10 a 12 anos.

O desempenho na natação pode ser determinado não somente por fatores fisiológicos, mas também por fatores técnicos, táticos, fatores intrínsecos de cada atleta, ambientais, etc. Consequentemente, tornam-se extremamente necessários, estudos que possam confirmar ou refutar a hipótese inicial da Velocidade Crítica a 4

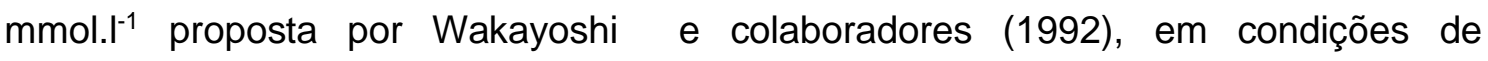
treinamento e atletas brasileiros.

\section{MATERIAL E MÉTODOS}

Este trabalho é resultado da Dissertação de Mestrado do autor Marcelo Celestino Vaccari (VACCARI, 2006).

\subsection{Caracterização da amostra}

O estudo desenvolveu-se com a equipe de natação FADENP da cidade de são josé dos campos/SP. Participaram da pesquisa 19 nadadores, com idade entre 16 e 30 anos, com envergadura variando entre $159 \mathrm{~cm}$ e $199 \mathrm{~cm}$, peso variando entre $49 \mathrm{~kg} \mathrm{e}$ $83 \mathrm{~kg}$ e a altura entre $154 \mathrm{~cm}$ e $192 \mathrm{~cm}$, sendo 8 indivíduos do sexo feminino (5 velocistas e 3 fundistas) e 11 indivíduos do sexo masculino (9 velocistas e 2 fundistas). 
Após assinatura do termo de informação e consentimento, os nadadores de elite da equipe de natação FADENP foram submetidos aos protocolos específicos de avaliação. O Presente trabalho foi submetido e aprovado pelo Comitê de ética em Pesquisa da Universidade do Vale do Paraíba.

\subsection{Critérios de exclusão}

Foram excluídos do estudo indivíduos com histórico recente (menos de 06 meses) de lesão musculoesquelética ou doenças respiratórias.

\subsection{Procedimentos}

\subsubsection{Protocolo de aquecimento}

Cada voluntário realizou um aquecimento em piscina que consistiu em natação livre de distância de 600 metros conforme treinamento padrão da equipe.

\subsubsection{Protocolo de avaliação}

Nadadores velocistas foram submetidos a protocolos de teste consistindo em 24 seqüências (tiros) de $100 \mathrm{~m}$, com intervalos de 30 segundos, totalizando 2.400 metros. Nadadores fundistas foram submetidos a protocolos de teste consistindo de 6 seqüências (tiros) de $400 \mathrm{~m}$, totalizando também, $2400 \mathrm{~m}$. Todos os testes foram realizados na mesma piscina de 25 metros. Foram realizadas 2 baterias de teste iniciais, com intervalo de 01 semana entre os testes, para aferição do protocolo. Foi solicitado que o atleta realizasse a natação do percurso em intensidade preconizada para acúmulo de $4 \mathrm{mmol}$ de lactato plasmático.

\subsection{Cuidados adotados para a realização dos testes}

O teste, de preferência, deve ser realizado após o término de um bloco de treinamento, visando o diagnóstico qualitativo da capacidade aeróbia e anaeróbia referente à fase do treinamento previamente proposto pelo técnico.

Deve ser realizado na semana regenerativa. Sem interferência de trabalho intenso anaeróbio (somente após 72 horas). O atleta deve encontrar-se descansado. Os testes subseqüentes também devem ser realizados no mesmo horário e mesmo piscina (distância) dos anteriores para ter validade comparativa.

\subsection{Sequência para realização do teste:}

a) Preenchimento da tabela auxiliar da capacidade aeróbia e anaeróbia. 
b) Anotar a freqüência cardíaca (FC) basal (pela manhã, ao acordar), determinar a freqüência cardíaca máxima do atleta, verificar a FC pré-teste do atleta (importante para se calcular a medida do percentual do VO2 máx do teste).

c) O tiro dos 400 metros é realizado preferencialmente no estilo livre, mas também pode ser realizando no estilo principal do atleta (peito e costas) conforme a preferência do técnico/atleta. O nadador do estilo borboleta deve realizar o teste no estilo livre. O nadador de medley pode também ser realizado no seu melhor estilo.

d) O aquecimento deve ser o suficiente para tornar o atleta apto para a realização do teste.

e) Tiro dos 400 metros (duração de 4.5 a 6 minutos) tem que ter duração suficiente para que a produção e eliminação do lactato alcance quantidades equilibradas. $O$ nado deve ser à velocidade moderada e constante durante as quatro passagens dos 100 metros. A produção de lactato pelo músculo cessa após o tiro, assim as concentrações plasmáticas começam imediatamente a diminuir.

f) O tiro tem como finalidade medir a capacidade aeróbia do atleta, portanto, deve ser sempre realizado abaixo do limiar anaeróbio. Atletas velocistas, pela alta capacidade de produção de lactato, devem realizar o tiro entre $50-60 \%$ da velocidade máxima prevista. Os meio fundistas, entre $60-70 \%$ da velocidade máxima prevista. Já os atletas fundistas, pela sua alta capacidade de remoção e, baixa a média capacidade de produção de lactato, entre $80-90 \%$ da velocidade máxima prevista.

g) Tanto a produção quanto a eliminação do lactato estão em perfeito estado de equilíbrio dinâmico. O lactato deve ser coletado imediatamente após o teste; 1 minuto após o término do tiro.

h) No caso de concentração do lactato no sangue, após a coleta aos três minutos, esteja acima do primeiro minuto, o teste, em princípio, deve ter sido realizado a uma velocidade acima do limiar anaeróbio, portanto, o resultado da capacidade aeróbia pode ser falso (pode acontecer com velocistas e meio fundistas que, durante as passagens sem conhecimento de ritmo podem acelerar nos últimos 100 metros).

i) Neste caso o teste deve ser repetido após os seguintes procedimentos; o atleta nada durante 15 minutos 30 a 40\% do esforço máximo (regenerativo), a seguir, 5 a 10 minutos de repouso absoluto na borda até que seu pulso e respiração voltem ao seu ritmo do repouso. Repetir o tiro num ritmo constante à intensidade compatível conforme a finalidade do teste e proceder a coleta após o tiro.

j) Anotar tempo (em segundos), ciclo de braçada a cada 50 metros, pulso, imediatamente e 1 minuto após. 
O teste do cálculo do V4 dos 400 metros visa encontrar a intensidade do nado para o treinamento para séries com metragens de 400, 200 e 100 metros com intervalo entre os tiros de 10 e 30 segundos. Estas séries devem, no mínimo, abranger uma distância total de 2400 metros.

\subsection{Coleta sanguínea}

Após assepsia do dedo indicador da mão direita do atleta com Cloreto de Benzalcônio, foram utilizadas lancetas descartáveis (Roche Diagnostics) para a realização de um pequeno furo. Foi coletada uma única gota de sangue em fita específica para teste de lactato plasmático (Roche Diagnostics), e então levado ao aparelho Accutrend Lactate (Roche Diagnostics) para determinação automatizada das concentrações de lactato plasmático após 1 e 3 minutos do final do esforço de nado.

Como o lactato se movimenta do músculo para a circulação sanguínea, onde é finalmente medido, ocorre um atraso entre tempo do pico da concentração do lactato muscular alcançada e o tempo dos valores mais altos de lactato no sangue. Portanto, para certificarmo-nos a respeito dos valores de pico da concentração de lactato sanguínea após o exercício, foram realizadas duas coletas de sangue.

\subsection{Análise estatística}

Foram calculadas as médias do tempo de nado e lactato plasmáticos obtidos de todos os atletas e comparadas as médias preconizadas pelos pré-testes do protocolo do V4 utilizando-se o teste t-student para amostras pareadas. Foram considerados significantes valores de $p<0,05$.

\section{RESULTADOS}

\subsection{Tempo preconizado $X$ tempo realizado}

O primeiro fator limitante do protocolo do V4 é a capacidade de reproduzir a velocidade de natação adequada para a obtenção do tempo preconizado para obtenção das concentrações plasmáticas de lactato desejadas.

Com relação à realização correta deste parâmetro, em nosso estudo os atletas foram capazes de desenvolver a velocidade de natação adequada, ou seja, não foram observadas diferenças significativas com relação ao tempo gasto para realização dos testes práticos, tanto para os nadadores velocistas quanto para fundistas (Figuras $1 \mathrm{e}$ 2) 
Figura 1 - A figura ilustra o Tempo proposto calculado pelo protocolo V4 e o tempo realmente realizado pelos atletas velocistas.

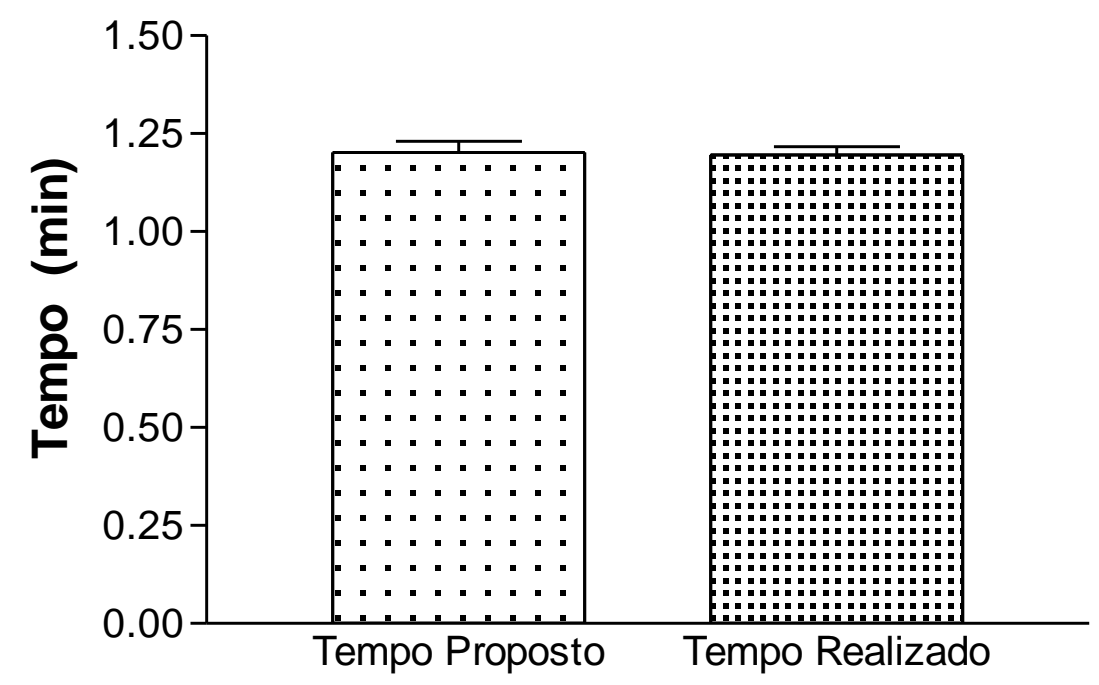

Figura 2 - A figura ilustra o tempo proposto calculado pelo protocolo V4 comparado ao tempo realmente realizado pelos atletas fundistas.

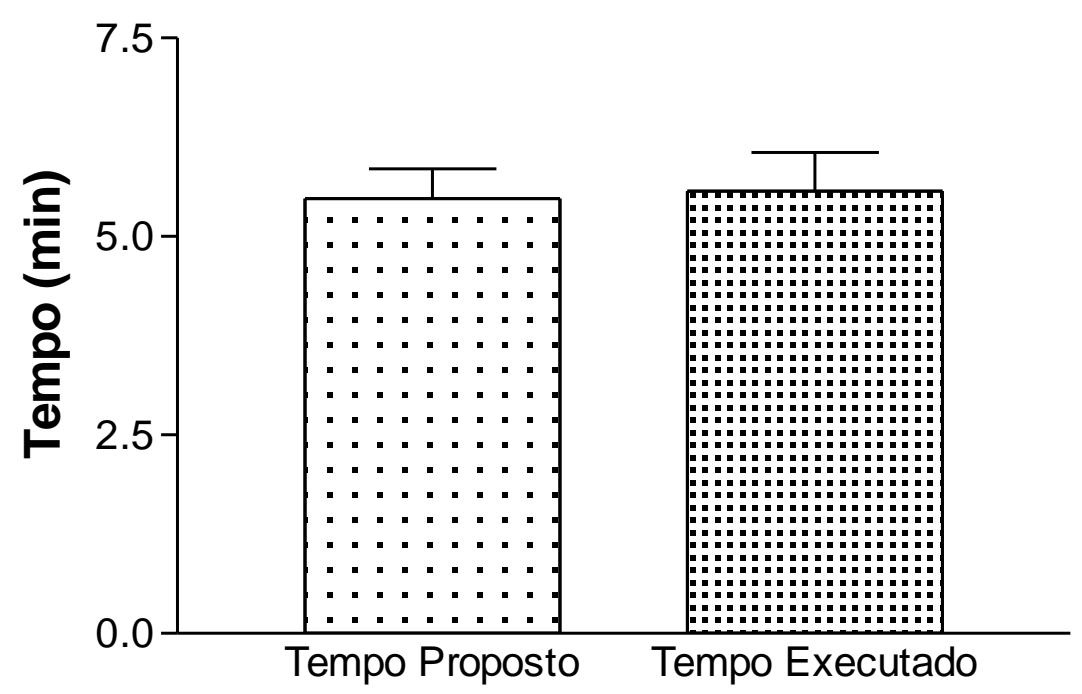

\subsection{Lactato preconizado X lactato obtido}

Tendo em vista o cumprimento adequado da meta preconizada com relação ao tempo de natação, observamos então as concentrações obtidas de lactato plasmático comparados com aquelas concentrações preconizadas. Pudemos observar que não houveram diferenças significativas para as concentrações de lactato plasmático nos atletas velocistas (Figura 3). No entanto, quando analisamos os resultados das concentrações de lactato plasmático dos nadadores fundistas, pudemos observar que, 
em todos os atletas não foram alcançadas as concentrações preconizadas teóricas. Havendo diferenças estatisticamente significantes $(p<0.05)$ entre as concentrações preconizadas e aquelas obtidas no teste prático (Figura 4).

Figura 3 - A figura ilustra as concentrações de Lactato plasmático preconizado calculado pelo protocolo V4 e as concentrações de lactato plasmático obtidas pelos atletas velocistas, após a aferição do tempo proposto e do tempo realizado.

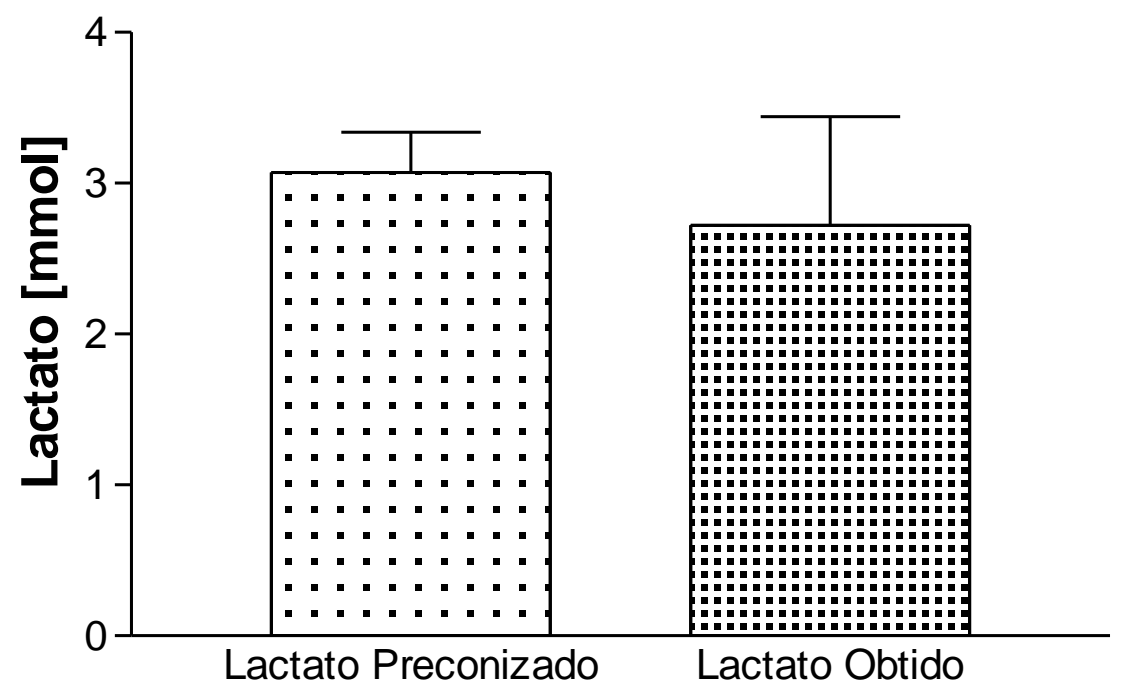

Figura 4 - A figura ilustra as concentrações de Lactato plasmático preconizado calculado pelo protocolo V4 e as concentrações de lactato obtidas pelos atletas fundistas, após a aferição do tempo proposto e do tempo realizado.

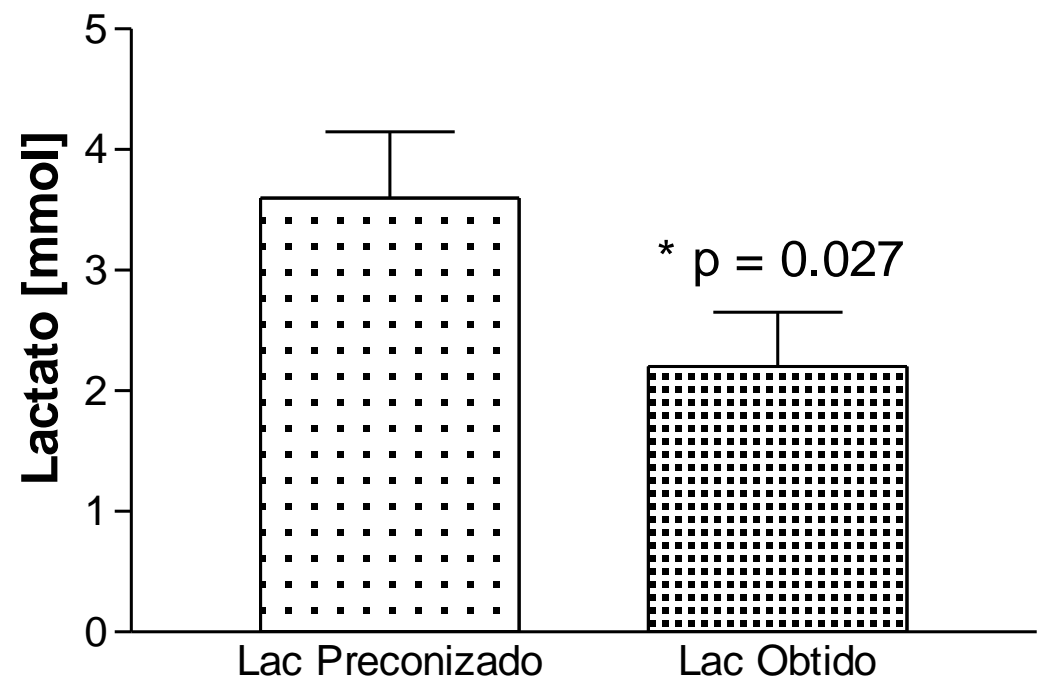




\section{DISCUSSÃO}

Análises precisas e a avaliação dos componentes da performance competitiva no contexto do treinamento desportivo constituem processos importantes para treinadores e cientistas do esporte, tornando-se ferramentas obrigatórias em programas de treinamento e competições. Há algum tempo o esporte de alto rendimento deixou de ser uma atividade empírica para se tornar, cada vez mais, uma verdadeira ciência. Protocolos diagnósticos visando treinamento e desempenho, devem prover bases confiáveis para a análise de resultados e tendências decorrentes do treinamento, assim como para predição de performances competitivas futuras (SMITH; NORRIS; HOGG, 2002).

Alguns aspectos do treinamento vêm assumindo especial importância, como a maior velocidade de curta duração alcançada, a velocidade na potência aeróbica máxima, velocidade no estado de equilíbrio fisiológico, economia de nado e capacidade anaeróbica. Além disso, aspectos biomecânicos e fisiológicos também estão intimamente relacionados ao desempenho (ATKINSON; REILLY, 1996; HUOTMARCHAND et al, 2005).

Neste trabalho, nós avaliamos o protocolo do V4 com o objetivo de realizar um teste prático para confirmação de sua capacidade preditiva em nadadores com diferentes características, ou seja, velocistas e fundistas. Devido à falta de trabalhos específicos sobre o assunto, muitas vezes os treinadores lançam mão de protocolos padronizados, mas sem a devida comprovação de sua fidedignidade para diferentes fins. Subjetivamente, podemos supor que nadadores com diferentes características fisiológicas, de nado ou mesmo antropométricas, possam responder de maneiras diferentes a um mesmo teste de esforço. Com efeito, nadadores de provas de longa distância que cumpriram o tempo preconizado no teste de esforço proposto obtiveram uma concentração de lactato plasmática significativamente abaixo daquela preconizada pelos cálculos matemáticos, após os testes iniciais de calibração, podemos supor que este fato ocorreu devido a intensidade do nado aplicado e pelo nível de condicionamento físico dos atletas, os quais pelas características do nado de longa distância suportam uma concentração maior de lactato (Figura 4). Por outro lado, nadadores velocistas apresentaram alta fidedignidade entre as concentrações de lactato preconizadas matematicamente pelo teste, quando realizaram o teste de esforço no tempo pré-determinado.

Toubekis, Tsami e Tokmakidis (2006) observaram em nadadores infantis e juvenis, que a velocidade crítica pode ocorrer abaixo do V4, e que a inclusão de testes de longas distâncias podem subestimar a velocidade crítica. Isto pode ser atribuído a não linearidade da curva tempo vs. distância observada na natação (WRIGHT; SMITH, 1994; DEKERLE et al, 2002). Neste sentido, tais observações podem justificar o porquê do teste aplicado neste trabalho ter apresentado bom resultado para nadadores velocistas, porém não tendo se mostrado adequado para nadadores de 
distâncias mais longas em testes de 400 m.

Recentemente, Ferreira et al (2015) utilizou o protocolo do V4 para avaliação de performance de atletas Masters para comparar diferenças entre gênero, biomecânica e bioenergética, concluindo que a performance parece estar mais relacionada a aspectos técnicos do que os que foram testados no estudo.

Toubekis et al (2011) examinaram a eficácia da velocidade crítica de nado para prescrição de treinamento e monitorou as mudanças induzidas na resistência aeróbia após um período de aumento do volume de treinamento em jovens nadadores. Neste estudo os autores sugerem que o ritmo de nado pode ser efetivamente utilizado para a melhora da resistência aeróbica em jovens nadadores. Os índices de resistência aeróbia usados para a avaliação da progressão dos nadadores mostraram diferentes taxas de mudança como resposta ao mesmo estímulo de treinamento e não podem ser usados de forma intercambiável para o planejamento do treinamento. Tais achados parecem estar de acordo com os resultados obtidos por nosso grupo.

Existem ainda pouco trabalhos no que diz respeito a validação do Teste do V4, amplamente utilizado na natação brasileira, e nossos resultados demonstram a necessidade de novos trabalhos que venham a melhor investigar a aplicabilidade do teste com diferentes populações com características fisiológicas e de treinamento distintas. No entanto, em nosso trabalho e sob nossas condições experimentais podemos concluir que, ao menos para nadadores velocistas os parâmetros preconizados pelo teste do V4 foram altamente fidedignos.

No presente estudo, sob as condições experimentais testadas, podemos concluir que para nadadores velocistas os parâmetros preconizados pelo teste do V4 foram altamente fidedignos. Contudo, o mesmo não se aplica a nadadores fundistas.

\section{REFERÊNCIAS}

ATKINSON, G.; REILLY, T. Circadian variation in sports performance. Sports Med., v. 21, n. 4, p. 292-312, 1996.

COYLE, E. F.; HOPPER, M. K.; COGGAN, A. R. Maximal oxygen uptake relative to plasma volume expansion. Int J Sports Med., v. 11, n. 2, p. 116-119, 1990.

DEKERLE, J. et al. Velocity and reliability of critical speed, criticalstroke rate and anaerobic capacity in relation to front crawl swimming performance. Int $\mathbf{J}$ Sports Med., v. 23, n. 2, p. 93-98, 2002.

DENADAI, B. S.; GRECO, C. C.; TEIXEIRA, M. Blood Lactate response and critical speed in swimmers aged 10-12 years of different standards. J Sports Sci., v. 18, n. 10, p.779-784, 2000.

FERREIRA, M. I. et al. Effect of Gender, Energetics, and Biomechanics on Swimming Masters Performance. J Strength Cond Res., v. 29, n. 7, p. 1948-1955, 2015.

GOLLNICK, P. D. et al. The effect of high-intensity exercise on the respiratory capacity 
of skeletal muscle. Pflugers Arch., v. 415, n. 4, p. 407-413, 1990.

HAWLEY, J. A.; STEPTO, N. K. Adaptations to training in endurance cyclists: implications for performance. Sports Med., v. 31, n. 7, p. 511-520, 2001.

HAY, J.G.; GUIMARÃES, A. C. A quantitative look at swimming biomechanics.

Swimming Tech., v. 20, n. 1, p. 11-17, 1983.

HECK, H. et al. Justification of the 4-mmol/L lactate threshold. Int J Sports Med., v. 6, n. 3, p. 117-130, 1985.

HUOT-MARCHAND, F. et al. Variations of stroking parameters associated with $200 \mathrm{~m}$ competitive performance improvement in top-standard front crawl swimmers. Sports Biomech., v. 4, n. 1, p. 89-99, 2005.

MADSEN, O. Untersuchungen uber Einflubgroben auf Parameter des

Energiostoffwechsels beim freien kraulscheimmen, Dissertation (Master) - Dtsch. Sporthochschule Koln, 1982.

MORTON, R. H. Modeling training and overtraining. J Sports Sci., v. 15, n. 3, p. 335340, 1997.

NELSON, R. C.; BROWN, P. L.; KENNEDY, P. W. An analysis of Olympic swimmers in the 1988 Summer Games. State College (PA): Pennsylvania State University, 1990.

OLBRECHT J. The Science of Winning: planning, periodizing and optimizing swim training. London: Swimshop Lutom, 2000.

OLBRECHT, J. et al. Relationship Between Swimming Velocity and Lactic Concentration During Continuous and Intermittent Training Exercises. Int J Sports Med., v. 6, n. 2, p. 74-77, 1985.

PANSOLD, B.; ZINNER, J. Selection, analysis and validity of sportspecific and ergometric incremental test programmes. In: Bachl N, Graham TE, Löllgen H, editors. Advances in ergometry. Berlin: Springer-Verlag; p. 180-214, 1991.

PYNE, D.; MAW, G.; GOLDSMITH, W. Protocols for the physiological assessment of swimmers. In: Gore C, editor. Physiological tests for elite athletes. Champaign (IL): Human Kinetics, p. 372-382, 2000.

SJODIN, B.; JACOBS, I.; SVENDENHAG J. Changes in the onset of blood lactate accumulation (OBLA) and muscle enzymes after training at OBLA. Eur J Appl Physiol., v. 49,n. 1, p. 45-57, 1982.

SMITH, D. J.; NORRIS, S. R.; HOGG, J. M. Performance Evaluation of Swimmers: Scientific Tools. Sports Med., v. 32, n.9, p. 539-554, 2002.

STEGMAN, H.; KINDERMANN, W.; SCHNABEL, A. Lactate kinetics and individual anaerobic threshold. Int J Sports Med., v. 2, n. 3, p. 160-165, 1981.

TEGTBUR, U.; BUSSE, M. W.; BRAUMANN, K. M. Estimation of an individual equilibrium between lactate production and catabolism during exercise. Med Sci Sports Exerc., v. 25, n. 5, p. 620-627, 1993.

TOUBEKIS, A. G. et al. Training-induced changes on blood lactate profile and critical 
velocity in young swimmers. J Strength Cond Res., v. 25, n. 6, p. 1563-1570, 2011.

TOUBEKIS, A. G.; TSAMI, A. P.; TOKMAKIDIS, S. P. Critical velocity and lactate threshold in young swimmers. Int J Sports Med., v. 27, n. 2, p. 117-123, 2006.

TROUP, J. P. Setting up a season using scientific training. Swimming Tech, v. 23, n. 1, p 8-16, 1986.

VACCARI, M. C. Estudo de Aferição do Protocolo de Velocidade de Nado para 4 mmol.-1 de Lactato (Protocolo do V4) para nadadores. 2006. 47f. Dissertação (Mestrado) - Universidade do Vale do Paraíba, São José dos Campos, 2006.

WAKAYOSHI, K. et al. Determination and validity of critical velocity as an index of swimming performance in the competitive swimmer. Eur J Appl Physiol., v. 64, n. 2, p. 153-157, 1992.

WELTMAN, A. The Blood Lactate Responses to Exercise. Champaing (IL): Human Kinetics, 1995.

WRIGHT, B.; SMITH, D. A protocol for the determination of critical speed as an index of swimming endurance performance. In: MIYASHITA, M.; MUTOH, Y.; RICHARDOSN, A. editors. Medicine and Science in Aquatic Sports. Basel: Karger; p.55-59, 1994. 